\title{
The bovine colostrum microbiome and its association with clinical mastitis
}

\author{
Svetlana F. Lima, ${ }^{*}$ Andre G. V. Teixeira, ${ }^{*}$ Fabio S. Lima,† Erika K. Ganda, ${ }^{*}$ Catherine H. Higgins, ${ }^{*}$ \\ Georgios Oikonomou, ${ }^{\star} \ddagger$ and Rodrigo C. Bicalho*1 \\ *Department of Population Medicine and Diagnostic Sciences, Cornell University, Ithaca 14853, New York \\ †Department of Veterinary Medical Science, College of Veterinary Medicine, University of Illinois, Urbana 61802 \\ fDepartment of Epidemiology and Population Health, Institute of Infection and Global Health, University of Liverpool, Neston CH64 7TE, \\ United Kingdom
}

\section{ABSTRACT}

In an effort to characterize colostrum microbial diversity and its potential associations with early-lactation clinical mastitis, we used high-throughput sequencing of the $16 \mathrm{~S}$ rRNA gene to investigate the bovine colostrum microbiome. A prospective observational study was conducted that included 70 Holstein cows; colostrum samples were collected from all 4 mammary gland quarters. Colostrum samples were categorized according to whether the quarter was diagnosed (CMC) or not diagnosed (NCMC) with clinical mastitis during the first $30 \mathrm{~d}$ postpartum. Colostrum samples were dominated by Firmicutes, Bacteroidetes, Proteobacteria, Actinobacteria, Fusobacteria, and Tenericutes phyla, with the 6 most common taxa [order (o), family (f), and genus (g)] being g_Staphylococcus, g_Prevotella, f_Ruminococcaceae, o_Bacteroidales, o_Clostridiales, and g_Pseudomonas. The colostrum microbiota of primiparous cows was significantly richer (higher number of bacterial species) than that of multiparous cows, and differences in colostrum taxonomic structure between parities were also observed. The microbial community of NCMC samples of primiparous cows was significantly more diverse than that of CMC samples, and the relative abundances of the Tenericutes and Fusobacteria phyla as well as the Mycoplasma and Fusobacterium genera were significantly higher in NCMC than in CMC samples of primiparous cows. The colostrum core microbiome, defined as the bacterial taxa common to all colostrum samples examined, was composed of 20 taxa and included bacterial genera already known to be associated with mastitis (e.g., Staphylococcus, Mycoplasma, and Streptococcus spp.). Our results indicate that the colostrum microbiome of primiparous cows differs from that of multiparous cows, and it harbors some diversity and taxonomic markers of mammary gland health specific to primiparous cows only.

Received June 13, 2016.

Accepted December 13, 2016.

${ }^{1}$ Corresponding author: rcb28@cornell.edu
Key words: colostrum, mastitis, microbiome

\section{INTRODUCTION}

Colostrum is a complex biological fluid that supports the growth and health of the neonate (Ogra et al., 1977; Chase et al., 2008; Hammon et al., 2013) and plays a key role during this initial and critical period of life. Bovine colostrum (BC) is not only nutrient rich, it also contains growth factors and antimicrobial agents (Pakkanen and Aalto, 1997; Elfstrand et al., 2002) and harbors apparently beneficial microflora, such as members of the Bifidobacterium and Lactobacillus genera (Lindner et al., 2011), that are widely used as probiotics. Culture-independent microbiome studies are greatly improving our understanding of the microbial diversity of different bodily environments, such as human or bovine milk (Cabrera-Rubio et al., 2012; Kuehn et al., 2013; Ward et al., 2013; Oikonomou et al., 2014). Indeed, studies in humans have described the microbial composition of breast milk during lactation (CabreraRubio et al., 2012; Ward et al., 2013; Khodayar-Pardo et al., 2014) and its potential effects on infant health (Ward et al., 2013). Human colostrum in these studies was also shown to harbor a diverse microbial community that was different from that of milk. Our group previously characterized the bovine milk microbial community and its relationship with mammary gland health (Oikonomou et al., 2012, 2014). However, only a few studies have investigated the microbial composition of bovine (Lindner et al., 2011) and human (CabreraRubio et al., 2012) colostrum. Lindner et al. (2011) isolated and sequenced 29 bacterial strains from BC samples; however, culture-independent characterization of the $\mathrm{BC}$ microbiome has not yet been conducted, and its potential effect on mammary gland health remains unknown.

In dairy cattle, colostrum is the first milk produced after a nonlactating period, commonly referred to as the dry period (Watters et al., 2008). The dry period is critical for replacement of senescent mammary epithelial cells as well as the elements produced by those cells 
(Capuco et al., 1997). Studies have shown an increased risk of mammary gland infection correlated with IMI at the beginning or end of the dry period (Aarestrup and Jensen, 1997; Green et al., 2002; Bradley and Green, 2004). Furthermore, DNA fingerprinting analysis demonstrated that the pathogens that cause infection in the dry period may persist, and that the infected udder quarter is more likely than the uninfected quarters to develop mastitis as a consequence of these dry period pathogens (Bradley and Green, 2000). Because significant numbers of IMI have been detected in both the dry period and the early fresh period, targeting new intervention procedures to these periods may help to improve udder health and milk production (Natzke, 1981). Thus, characterization of the BC microbial community could help identify the bacterial profiles closely associated with mammary gland infection soon after parturition.

Inflammation of the mammary gland parenchyma (mastitis) is mainly triggered by bacterial infection (Federation, 1987; Oviedo-Boyso et al., 2007). Bovine mastitis resulting from bacterial infection is a huge economic burden for the dairy industry worldwide. The estimated cost of each case of clinical mastitis (CM), which occurs in the first $30 \mathrm{~d}$ of lactation, is approximately $\$ 444$, including direct costs, such as therapeutics, nonsaleable milk, and cow death, and indirect costs, such as future milk production loss and premature culling (Rollin et al., 2015). The USDA National Animal Health Monitoring Service reported in 2007 that CM affects $94.9 \%$ of American dairy herds (USDA, 2007); additionally, the reported incidence of CM per American farm unit during the same study period was approximately 16.5\% (USDA, 2007). Thus, the aim of our study was to use metagenomic highthroughput sequencing of the $16 \mathrm{~S}$ rRNA gene to assess the microbial community of colostrum of Holstein dairy cows and evaluate its potential association with early CM.

\section{MATERIALS AND METHODS}

\section{Ethics Statement}

The research protocol was reviewed and approved by the Cornell University Institutional Animal Care and Use Committee (protocol number 2013-0056). The methods were carried out in accordance with the approved guidelines.

\section{Study Design and Study Population}

We used a prospective observational cohort study design in which colostrum samples were aseptically col- lected daily (morning and afternoon) at the first milking within $8 \mathrm{~h}$ after parturition on a large commercial dairy farm situated in upstate New York. After colostrum sample collection, the cohort of animals was followed for $30 \mathrm{~d}$ postpartum for mammary gland health characterization. Colostrum samples were obtained from each mammary gland quarter, defined as left front (LF), left hind $(\mathrm{LH})$, right front $(\mathrm{RF})$, and right hind $(\mathrm{RH})$ cow udder quarters. All postpartum cows that were first milked within $8 \mathrm{~h}$ after parturition from November to December of 2013 were eligible to be enrolled in this study, and a total of 100 Holstein dairy cows were sampled at first milking. After sample exclusion due to absence of cow health report, missed colostrum sample collection due to udder-quarter trauma or structural disorder, or problems with downstream screening (e.g., failed DNA amplification or poor sequencing quality), a total of 280 samples from 70 cows was submitted for microbiome analysis. In the present study, all 280 colostrum samples presented the same physical appearance (yellow hue color, high fat content, and no sign of inflammation or trauma, such as blood, flakes, or clot).

\section{Animals and Facilities}

The commercial dairy farm involved in the study milked 3,450 Holstein cows thrice daily in a double 52-stall parallel milking parlor. During the study period, the average milk production per cow per day was $40 \mathrm{~kg}(\mathrm{SE}=4.41)$, and the incidence of mastitis was $\mathbf{1 9 . 2 \%}$. Following the farm's protocol, lactating pregnant cows were submitted to a dry period of $60 \mathrm{~d}$ and, at dry off, multiparous cows received intramammary antibiotic therapy (Orbenin-DC, Merck Animal Health, Kenilworth, NJ). Pregnant heifers and cows were housed together in freestall barns with concrete stalls covered with rubber mattresses and bedded with dried manure solids, separated from the lactating cows. Pregnant animals were housed into 2 separate groups: the far-off group (where dry cows remained until $2 \mathrm{wk}$ before expected calving), and the close-up group (cows in their last 2 wk before expected calving). Pregnant animals that were at stage 1 or 2 of parturition were moved into the deep-bedded maternity barn, with 4 identical group pens (a total of 3 cows per pen), to calve. Right after parturition, calves were removed from the maternity pen and placed into a newborn pen bedded with dry sawdust and heated with heating lamps. Fresh cows were first milked within $8 \mathrm{~h}$ of calving in the double 52-stall parallel milking parlor.

Prepartum heifers and cows were given a high-fiber, low-energy density diet, whereas postpartum cows were given a low-fiber content, high-energy density diet derived from higher starch and fat supplementation. 
The diet was formulated to meet or exceed the nutrient requirements for lactating Holstein cows weighing 650 $\mathrm{kg}$ and producing $45 \mathrm{~kg}$ of $3.5 \% \mathrm{FCM}$ (NRC, 2001).

\section{Sample Collection}

One member of our research group collected individual colostrum quarter samples aseptically at the first milking, within $24 \mathrm{~h}$ of parturition. Sampling methods followed standard recommendations by the National Mastitis Council's Laboratory Handbook on Bovine Mastitis (NMC, 1999). The first streams of colostrum from each quarter were discarded for mammary gland stimulation, and subsequently the teats were dipped in iodine tincture. The teats were then exposed to the disinfectant for $30 \mathrm{~s}$ and dried using an individual towel, as performed by the farm personnel. Research personnel performed the physical scrubbing of the teats with gauze soaked in $70 \%$ alcohol. Once more, the first streams of colostrum were discarded and the colostrum samples were then collected. A 10-mL aliquot of colostrum was collected in a sterile $50-\mathrm{mL}$ centrifuge tube (VWR International, Radnor, PA). Samples were kept on ice, transported to the laboratory, and stored at $-20^{\circ} \mathrm{C}$.

\section{Case Definition}

Clinical mastitis examination was performed at the milking parlor, thrice daily on all lactating cows, by farm employees with extensive experience and trained by Cornell University veterinarians (Ambulatory and Production Medicine Department). Fore-stripping was performed and quarters presenting abnormal milk (i.e., presence of flakes, clots, or serous milk), independently of systemic illness and signs of inflammation of the mammary gland, were diagnosed as CM.

\section{DNA Extraction}

Isolation of DNA from colostrum samples was performed by adapting the protocol of Rasolofo et al. (2010). A 10-mL aliquot of colostrum was clarified by the addition of $2 \mathrm{~mL}$ of $0.5 M$ EDTA at $\mathrm{pH} 8.0$, followed by centrifugation $\left(12,000 \times g\right.$ at $4^{\circ} \mathrm{C}$ for 30 min). The supernatant containing fat and protein was discarded and the cell pellet was washed twice with 1 $\mathrm{mL}$ of sucrose buffer (12\% sucrose, $25 \mathrm{mM}$ Tris- $\mathrm{HCl}$, $\mathrm{pH}$ 8.0) followed by centrifugation $\left(12,000 \times g\right.$ at $4^{\circ} \mathrm{C}$ for $30 \mathrm{~min}$ ). The pellet was resuspended in $0.4 \mathrm{~mL}$ of sucrose buffer containing $800 \mu \mathrm{g}$ of lysozyme and incubated for $1 \mathrm{~h}$ at $37^{\circ} \mathrm{C}$. Finally, total metagenomic DNA was extracted by using a Powersoil DNA Isolation Kit (Mo Bio Laboratories, Carlsbad, CA) following the manufacturer's protocol. The DNA concentration was evaluated by optical density using a NanoDrop ND-1000 spectrophotometer (NanoDrop Technologies, Rockland, DE) at wavelengths of 230, 260, and $280 \mathrm{~nm}$.

\section{PCR Amplification of the V4 Hypervariable Region of the Bacterial 16S rRNA Gene}

Amplification of the 16S rRNA gene was performed by PCR using barcoded primers on all metagenomic DNA samples of colostrum. For amplification of the V4 hypervariable region of the bacterial/archaeal $16 \mathrm{~S}$ rRNA gene, primers 515F and 806R were used according to previously described methods and optimized for the Illumina MiSeq platform (Caporaso et al., 2012). The Earth Microbiome Project (http://www.earthmicrobiome.org/; Gilbert et al., 2010) was used to select 140 different 12-bp error-correcting Golay barcodes for the procedure, as previously described (Caporaso et al., 2012). The 5'-barcoded amplicons were generated in triplicate using 12 to $300 \mathrm{ng}$ of template DNA, $2 \times$ EconoTaq Plus Green Master Mix (Lucigen, Middleton, WI), and $10 \mu M$ of each primer. The PCR conditions for amplification of the $16 \mathrm{~S}$ rRNA gene included an initial denaturing step of $94^{\circ} \mathrm{C}$ for 3 min followed by 35 cycles of $94^{\circ} \mathrm{C}$ for $45 \mathrm{~s}, 50^{\circ} \mathrm{C}$ for $1 \mathrm{~min}$ and $72^{\circ} \mathrm{C}$ for $90 \mathrm{~s}$, and a final elongation step of $72^{\circ} \mathrm{C}$ for 10 min. Replicate amplicons were pooled and purified using the Gel PCR DNA Fragment Extraction kit (IBI Scientific, Peosta, IA) and visualized by electrophoresis through $1.2 \%$ (wt/vol) agarose gels stained with 0.5 $\mathrm{mg} / \mathrm{mL}$ of ethidium bromide. Blank controls, in which no DNA were added to the reaction, were performed, and no amplification was detected. Purified amplicon DNA was quantified using the Quant-iT PicoGreen and dsDNA Broad Range Assay Kit (Life Technologies Corporation, Carlsbad, CA).

\section{Sequence Library Analysis}

Aliquots of colostrum amplicon samples were diluted to the same concentration and then pooled into 3 different runs according to individual barcode primers for the 16S rRNA gene. Final equimolar libraries were sequenced using the MiSeq reagent kit v2 (300 cycles) on the MiSeq platform (Illumina Inc., San Diego, CA). The 16S rRNA gene sequences generated were processed through the open source software pipeline Quantitative Insights Into Microbial Ecology (QIIME; version 1.7.0-dev; Caporaso et al., 2012). Sequences were filtered for quality using established guidelines (Bokulich et al., 2013). Sequences were binned into operational taxonomic units (OTU) based on 97\% identity using UCLUST (Edgar, 2010) against the Greengenes 
reference database (McDonald et al., 2012; May 2013 release). Low-abundance clusters were filtered and chimeric sequences were removed using USEARCH (Edgar, 2010). The representative sequences for each OTU were compared against the Greengenes database for taxonomy assignment, and only full-length, highquality reads $(\mathrm{r}=0)$ were used for analysis. The output of this workflow is a classification of reads at multiple taxonomic levels: kingdom (k), phylum (p), class (c), order (o), family (f), genus (g), and species (s).

Shannon diversity and Chaol indexes were calculated using the QIIME pipeline. Before estimating the Shannon and Chao1 indexes, all sample libraries were rarefied to an equal depth of 10,000 sequences using QIIME.

\section{Sample Categorization and Statistical Analysis}

Colostrum samples from mammary gland quarters that were diagnosed with $\mathrm{CM}$ in the first $30 \mathrm{~d}$ postpartum were categorized as clinical mastitic colostrum (CMC), and those samples from mammary gland quarters that were free of CM during the first $30 \mathrm{~d}$ postpartum were categorized as nonclinical mastitic colostrum (NCMC). Descriptive statistics for the total number of cows, quarters, and CM incidence (after applying the exclusion criteria) were assessed using the Distribution platform offered by JMP Pro 11 (SAS Institute Inc., Cary, NC). The Pearson chi-squared test was used to compare the incidence of mastitis between parities.

Sample richness was calculated by using the Chao 1 richness index, and diversity by using Shannon diversity index. The Chao 1 index is a nonparametric procedure that estimates the minimum richness (number of OTU) in a sample by using the number of rare OTU (singletons and doublets; Chao, 1984). The Shannon index is calculated based on both richness and evenness (how evenly the taxa are distributed in a sample; Zand, 1976). The Shannon index increases both when the number of species and evenness increases. To evaluate differences of the Chao 1 and Shannon indexes between the groups tested (primiparous vs. multiparous; CMC vs. NCMC), general linear models was fitted in JMP Pro 11. Two models were created: one with parity being the only independent variable (and which used colostrum samples only from quarters that did not experience $\mathrm{CM}$ during the first $30 \mathrm{~d}$ postpartum), and the other in which colostrum category (NCMC and CMC) blocked by parity was offered to the model as the only independent variable. The number of reads in each group tested (parity, colostrum category) was determined, and a similar procedure to the one described above was conducted.
Taxa data and relative abundance (proportion of each bacterial type within the total number of sequences in the sample) were used to describe the most abundant phyla and other OTU levels of the colostrum samples. To evaluate differences between microbial taxa of colostrum from primiparous and multiparous cows, colostrum samples only from quarters that did not experience CM during the first $30 \mathrm{~d}$ postpartum were used. A general linear model was fitted in JPM Pro 11. Parity, udder quarter, and the interaction between these terms were used as independent variables. Least squares means relative abundance and standard error of the mean for each bacterium were obtained.

Differences between colostrum categories (NCMC and $\mathrm{CMC}$ ) were also evaluated using general linear models fitted in JPM Pro 11 software. Microbial relative abundance (phyla and other OTU levels) in NCMC and $\mathrm{CMC}$, blocked by parity, were compared, and the least squares means relative abundance and standard error of the mean for each bacterium were obtained. A more detailed analysis of the differences in the relative abundances of g_Staphylococcus, g_Pseudomonas, g_Fusobacterium, g_Mycoplasma, g_Corynebacterium, g_Streptococcus, and g_Escherichia between colostrum categories, blocked by parity, was conducted using general linear models fitted in JPM Pro 11.

The core microbiome was defined as all taxa found to be present across all colostrum samples. A general linear model was performed and fitted in JMP Pro 11 to describe the least squares means relative abundance of the taxa shared by all colostrum samples of all primiparous and multiparous cows. The independent variable offered to the model was udder quarter category and blocked by parity.

\section{RESULTS}

\section{Sequencing Results, Descriptive Statistics, and a Diversity Indexes}

Sequences were filtered for size, quality, and for the presence of chimeras. The total post-quality-control number of sequences used in the study was $21,158,985$. The average coverage was 75,568 sequences per sample, with a standard deviation of 22,901 and a range of 7,517 to 151,326 . The number of sequences evaluated did not differ among parities, and colostrum categories (Supplemental Figure S1; https://doi.org/10.3168/ jds.2016-11604).

Descriptive statistics for the number of cows, number of quarters, and CM incidence are presented in Table 1. After applying the cow exclusion criteria, the incidence of CM at cow level for primiparous cows was $46.2 \%$ (n $=12)$ and for multiparous was $22.7 \%(\mathrm{n}=10)$. 

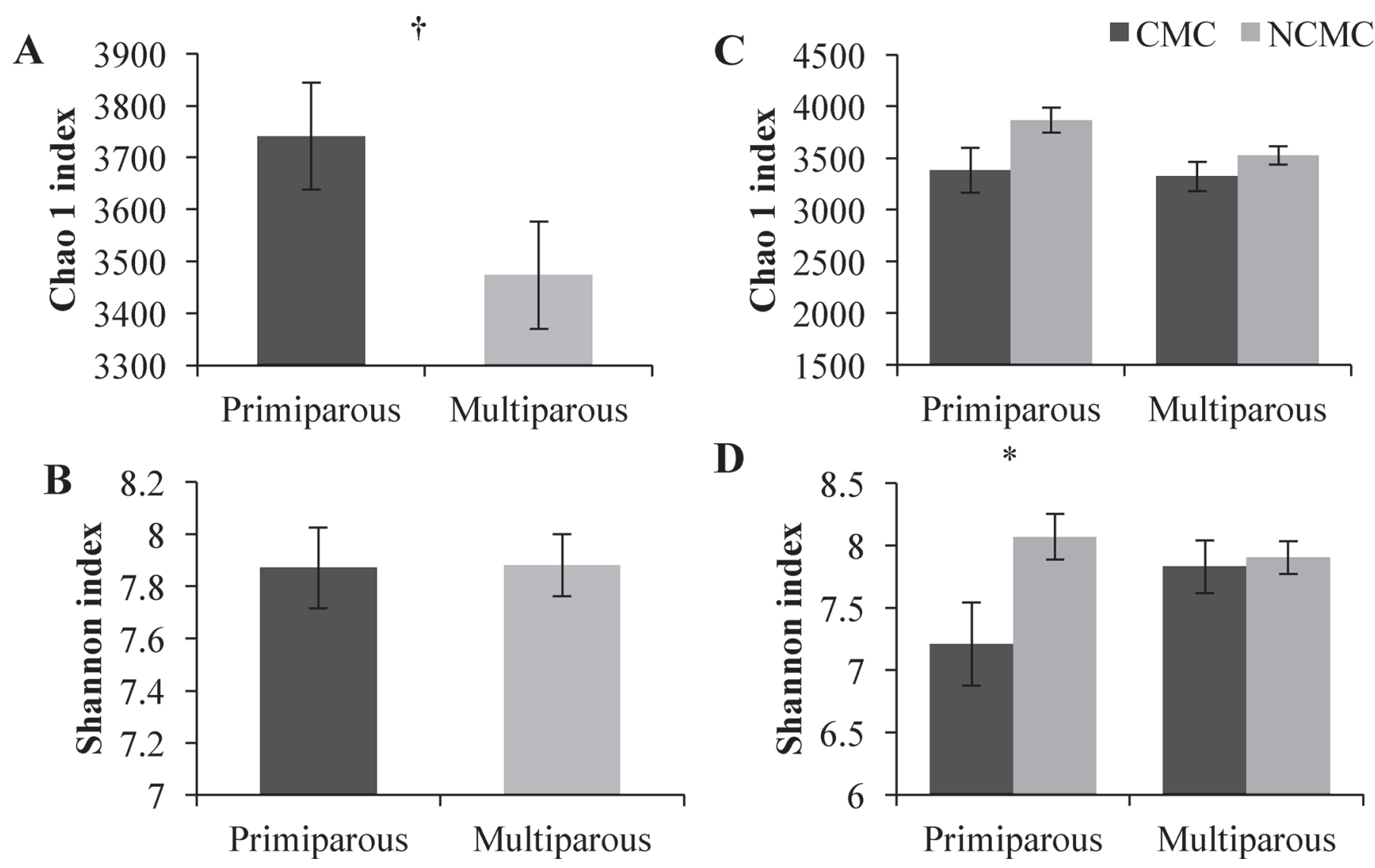

Figure 1. Chao 1 richness index and Shannon diversity index according to parity (A and B, respectively) and colostrum category within parity $(\mathrm{C}$ and $\mathrm{D}$, respectively). Error bars are positioned around the means and represent the SEM. A dagger $(\dagger)$ represents $0.05 \leq P<0.1$ and asterisk $(*)$ represents $P<0.05 . \mathrm{CMC}=$ clinical mastitic colostrum; NCMC $=$ nonclinical mastitic colostrum.

The Chao 1 richness and Shannon diversity indexes were calculated to obtain estimates of community diversity (Figures 1A-D). The Chao 1 index was higher in primiparous than in multiparous cows $(P<0.05$, Figure 1A), and tended to be higher in NCMC than in CMC samples of primiparous cows $(P=0.054$, Figure $1 \mathrm{C})$. The Shannon index was also higher for NCMC when compared with CMC samples of primiparous cows $(P<0.05$, Figure 1D). The Shannon index was not significantly different when parities were compared, nor when the colostrum categories of multiparous cows were compared (Figure $1 \mathrm{~B}$ and D, respectively). Similarly, the Chao 1 index did not differ significantly between the colostrum categories for multiparous cows (Figure 1C).

\section{Phylogenetic Profile and Core Microbiome}

Alignment of OTU at the $97 \%$ similarity threshold against the Greengenes database enabled identification

Table 1. Incidence of clinical mastitis during the first $30 \mathrm{~d}$ postpartum by cow and quarter for primiparous and multiparous cows used in the study; numbers in brackets indicate the number of quarters with clinical mastitis in each category, and $P$-value represents the difference between parities

\begin{tabular}{lccc}
\hline Item & Primiparous & Multiparous & $P$-value \\
\hline Total number of cows & 26 & 44 & - \\
Total number of quarters & 104 & 176 & - \\
Incidence of mastitis by quarter (\%) & $23.1(24)$ & $29.0(51)$ & 0.003 \\
Left front & $25.0(6)$ & $21.6(11)$ & 0.85 \\
Left hind & $20.8(5)$ & $27.5(14)$ & 0.25 \\
Right front & $25.0(6)$ & $27.5(14)$ & 0.43 \\
Right hind & $29.2(7)$ & $23.5(12)$ & 0.97 \\
\hline
\end{tabular}


of the colostrum bacterial community at the phylum and genus levels. Whereas most OTU were classified at the genus level, some were classified only at the phylum, class, order, or family level. The 6 most common bacterial phyla detected in colostrum samples from quarters that did not experience $\mathrm{CM}$ during the first 30 d postpartum are depicted in Figure 2A. Of the 6 most common phyla detected in both parities, the mean relative abundance (MRA) of the Firmicutes phylum was higher $(P<0.05)$ in samples of primiparous $(\mathrm{MRA}=$ $46.1 \%$; $\mathrm{SE}=1.8)$ than in multiparous cows $(\mathrm{MRA}=$ $40.8 \%$; SE $=1.6$ ), and the Fusobacteria phylum were lower $(P<0.05)$ in samples of primiparous (MRA $=$ $2.8 \%$; $\mathrm{SE}=0.6)$ than in multiparous cows $(\mathrm{MRA}=$ $4.8 \%$; $\mathrm{SE}=0.5$; Figure 2B).

The 15 most common OTU (order, family, and genus) detected in colostrum samples of primiparous and multiparous cows are shown in Figure $3 \mathrm{~A}$, and more detailed findings at the quarter level are presented in Figure 3B to E. Genus Bacteroides (MRA $=1.1$ and $2.5 \%, \mathrm{SE}=0.4$ and 0.3 for primiparous and multiparous, respectively; $P<0.05$ ), g_Acinetobacter (MRA $=2.3$ and $3.5 \%, \mathrm{SE}=0.5$ and 0.4 for primiparous and multiparous, respectively; $P<0.05$ ), g_Pseudomonas $(\mathrm{MRA}=3.5$ and $4.8 \%, \mathrm{SE}=0.5$ and 0.4 for primiparous and multiparous, respectively; $P<0.05$ ), and g_Fusobacterium (MRA $=2.8$ and $5.0 \%, \mathrm{SE}=0.6$ and 0.5 for primiparous and multiparous, respectively; $P=0.08)$ were either significantly higher or tended to be significantly higher in colostrum samples of multiparous cows than in primiparous cows (Figure 3A). Colostrum samples from primiparous cows had a higher abundance $(P<0.05)$ of g_Staphylococcus $(\mathrm{MRA}=$ 19.4 and $13.6 \%, \mathrm{SE}=1.9$ and 1.5 for primiparous and multiparous, respectively; $P<0.05)$ when compared with multiparous cows (Figure 3A). At the cow udder quarter level, microbial abundance differences between the parity samples were observed only in the right front and hind quarters (Figures 3D and E).

Microbial abundance differences at the phylum (Figures $4 \mathrm{~A}$ and $\mathrm{B}$ ) and other OTU levels (order, family, genus; Figures 5A and 5B) between the colostrum categories (NCMC and $\mathrm{CMC}$ ) were assessed. Among the most common phyla detected in the NCMC and CMC samples of primiparous and multiparous cows (Figure 4B), the relative abundances of the Tenericutes $(\mathrm{MRA}=5.6$ and $2.1 \%, \mathrm{SE}=1.4$ and 0.7 for $\mathrm{CMC}$ and NCMC, respectively) and Fusobacteria (MRA = 5.8 and $2.8 \%, \mathrm{SE}=0.9$ and 0.5 for $\mathrm{CMC}$ and $\mathrm{NCMC}$, respectively) phyla were higher $(P<0.05)$ in the CMC

\section{A}

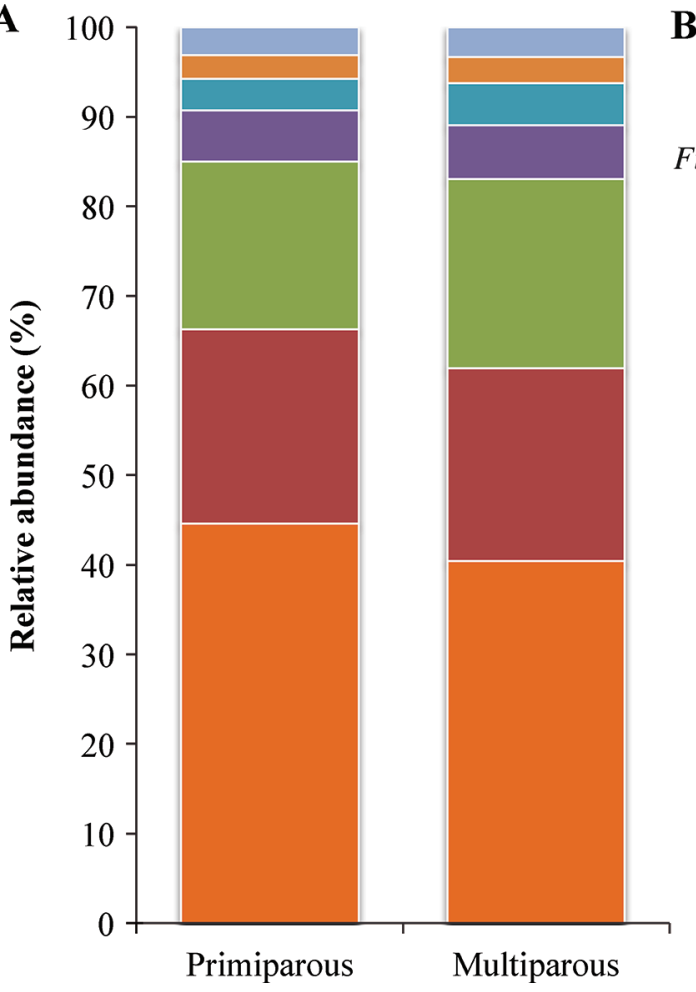

B

Multiparous $\square$ Primiparous

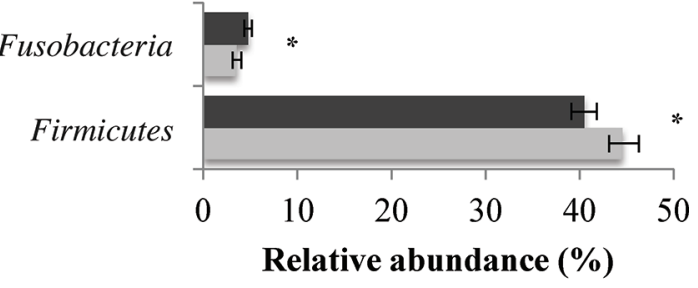

$\begin{array}{ll}\square \text { Firmicutes } & \square \text { Bacteroidetes } \\ \square \text { Proteobacteria } & \square \text { Actinobacteria } \\ \square \text { Fusobacteria } & \square \text { Tenericutes } \\ \square \text { Others } & \end{array}$

Figure 2. Aggregate microbiome composition at the phylum level for $16 \mathrm{~S}$ rRNA sequences of colostrum samples (A), and the mean relative abundance of Fusobacterium and Firmicutes phyla according to parity (B). Error bars are positioned around the means and represent the SEM. An asterisk $\left(^{*}\right)$ represents $P<0.05$. Color version available online. 
than in the NCMC samples of primiparous cows (Figure 4B). Descriptive cataloging of the 15 most common bacterial taxa (class, order, family, and genus) detected in colostrum samples of primiparous and multiparous cows according to each colostrum category and quarter evaluated is presented in Supplemental Figures S2A and S2B (https://doi.org/10.3168/jds.2016-11604). Genus Staphylococcus, g_Pseudomonas, g_Fusobacterium, g_Mycoplasma, g_Corynebacterium, g_Streptococcus, and g_Escherichia were subjected to more detailed analyses (Figure 5A and B). Genus Fusobacterium and g_Mycoplasma were relatively more abundant $(P$ $<0.05)$ in CMC than in NCMC samples of primiparous cows (Figure 5A), and g_Streptococcus tended to be more abundant in CMC than in NCMC samples of multiparous cows (Figure 5B).

The colostrum core microbiome, defined as the set of microbial organisms persistently present in all samples evaluated, regardless of parity or colostrum category, was assessed. In total, 20 bacterial taxa were detected in all colostrum samples from all quarters of all cows examined (Table 2).

\section{DISCUSSION}

To our knowledge, this is the first detailed description of the $\mathrm{BC}$ microbiome. Colostrum is the first milk following a long nonlactating period known as the dry period. The dry period is an interval of critical susceptibility to mammary gland infections (Green et al., 2002; Bradley and Green, 2004). Therefore, characterization of the $\mathrm{BC}$ microbiota may improve our understanding of the importance of the microbial community profile in early mastitis development. The results of our study indicate that colostrum harbors a rich and diverse microbial community, regardless of parity (primiparous or multiparous) or early lactation infection status (CMC or NCMC). Furthermore, we found differences in the bacterial taxonomic structure of colostrum between primiparous and multiparous cows, as well as between NCMC and CMC of primiparous cows.

Another interesting finding of our study is that the colostrum microbiota of primiparous cows was richer than that of multiparous cows. A possible explanation for this result is that these 2 groups are physiologi-
A

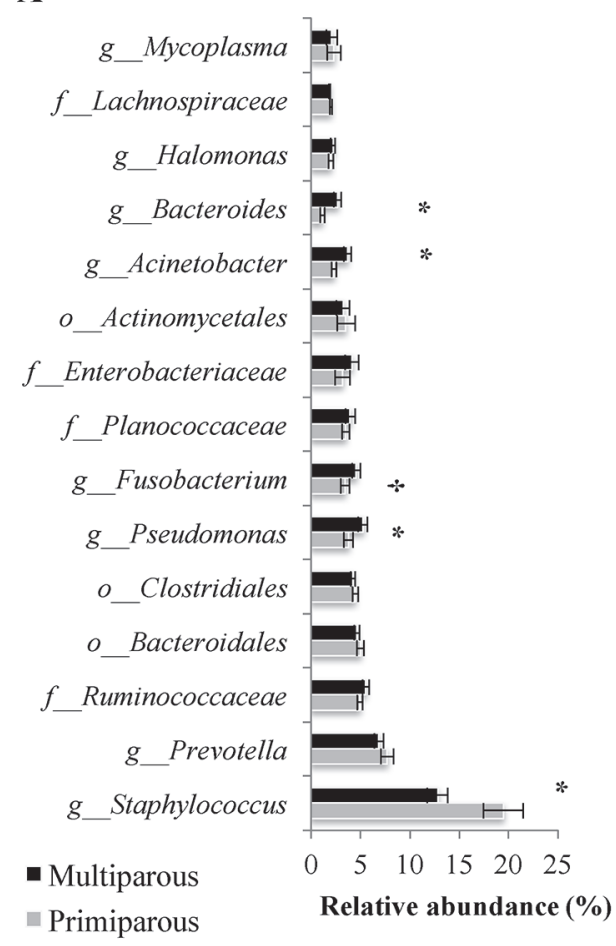

B

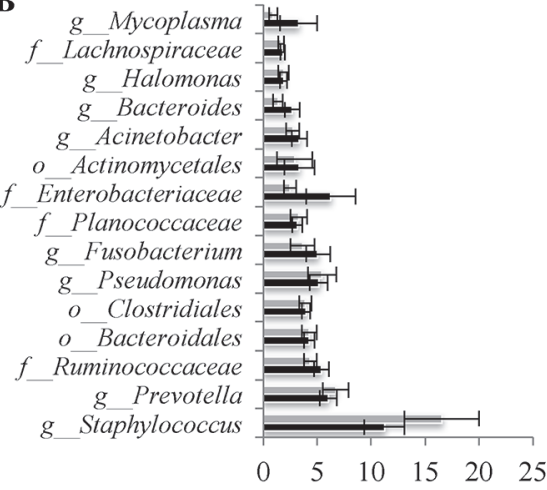

D



C

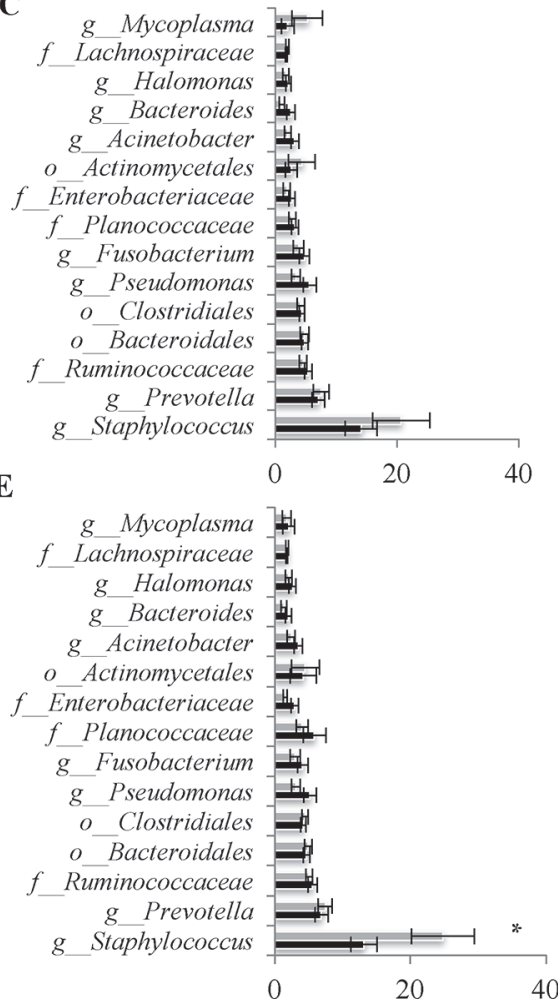

Figure 3. Bar graphs illustrating the 15 most common microbial taxa according to parity (primiparous and multiparous, A) and according to left front (B), left hind (C), right front (D), and right hind (E) cow udder quarters and parity. $o$ (order), $f$ (family), and $g$ (genus) represent the taxonomic level of the described bacterium. The mean relative abundance is represented by x-axis values. Error bars are positioned around the means and represent the SEM. A dagger $(\dagger)$ represents $0.05 \leq P<0.1$ and asterisk $(*)$ represents $P<0.05$. 
A

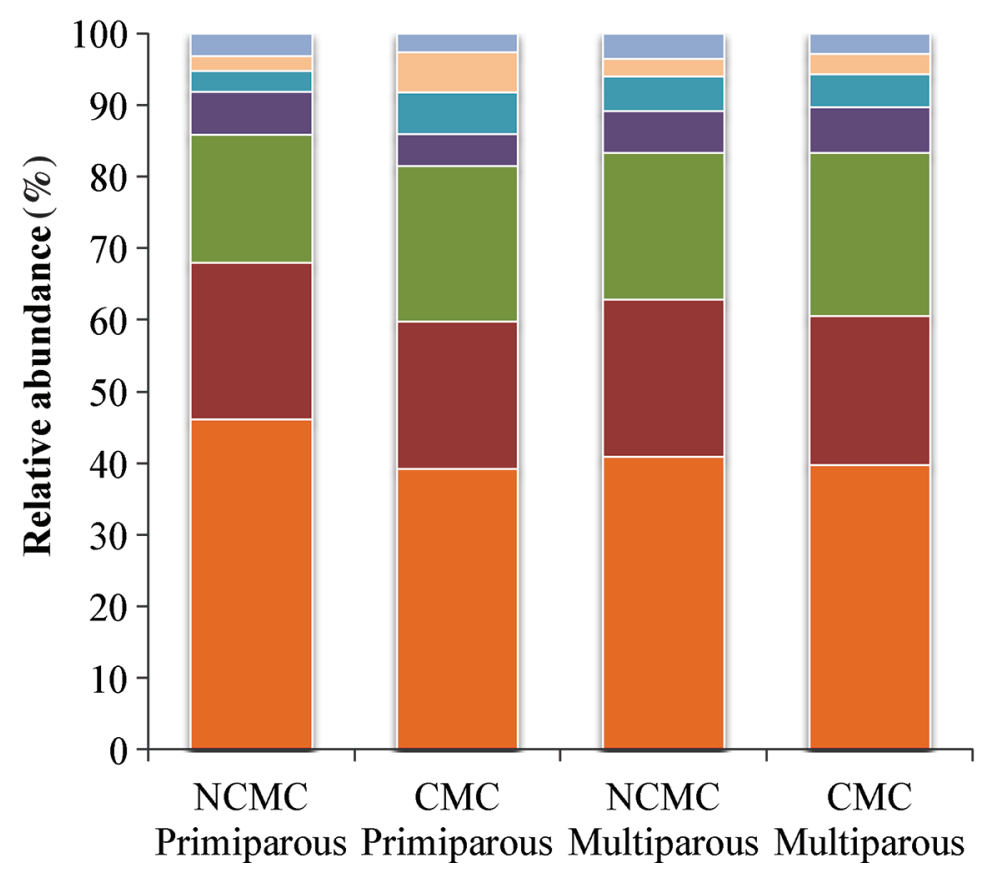

B

NCMC Primiparous

- CMC Primiparous

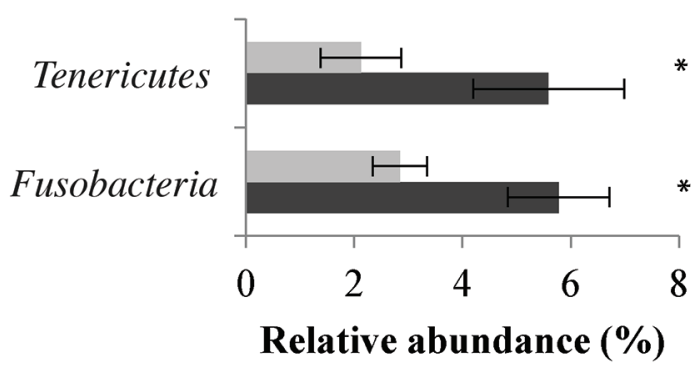

- Firmicutes

- Bacteroidetes

- Proteobacteria

- Actinobacteria

- Fusobacteria

$\square$ Tenericutes

Figure 4. Aggregate microbiome composition at the phylum level for $16 \mathrm{~S}$ rRA gene sequences of colostrum samples according to parity (primiparous and multiparous), and colostrum category [nonclinical mastitic colostrum (NCMC) and clinical mastitic colostrum (CMC); A] The mean relative abundance of the Tenericutes and Fusobacteria phyla in primiparous cows according to colostrum category (B). Error bars are positioned around the means and represent the SEM. An asterisk (*) represents $P<0.05$. Color version available online.

cally different; most obviously, the multiparous cows experienced at least 1 previous lactation, potentially including IMI events (Falentin et al., 2016). The use of dry period antibiotic therapy in multiparous cows is another plausible explanation for these findings. Although the intramammary antibiotic used at drying off in our study had a half-life of $4 \mathrm{wk}$, it could still have a lasting effect on the mammary gland and ultimately on the colostrum microbiome.

The OTU g_Staphylococcus, g_Prevotella, f_Ruminococcaceae, o_Bacteroidales, o_Clostridiales, and gPseudomonas were the 6 dominant taxa we identified in colostrum samples from primiparous and multiparous cows. In a recently published review, Addis et al. (2016) described the potential existence of an endogenous enteromammary pathway, in which intestinal bacteria migrate to the mammary gland, and this could explain the presence of gut bacteria such as g_Prevotella and f_Ruminococcaceae in colostrum samples (Addis et al., 2016). Furthermore, a limited microbiota of BC was previously described by Lindner et al. (2011), in which 29 strains, including Lactococcus casei, Staphylococcus pseudintermedius, Staphylococcus chromogenes, Bifidobacterium pseudolongum, and Propionibacterium acnes, were isolated from colostrum and identified by their 16S rRNA gene sequences (Lindner et al., 2011). The distinct bacterial organisms found by our group may be attributed in part to the methodology and technology we used. Whereas Lindner et al. (2011) extracted DNA from culture isolates followed by sequencing of the 16S rRNA gene, we opted for a culture-independent approach. Traditionally, the bacterial ecology of milk has been described by culture-dependent methods, which are time-consuming and limited in terms of their ability to detect a truly representative microbial community (Taponen et al., 2009; Oikonomou et al., 2012). In contrast, $16 \mathrm{~S}$ rRNA gene sequencing is a much more robust approach for determination of the complete bacterial community in an environment as well as taxon relative abundances (Amann et al., 1995; Schloss and Handelsman, 2005).

Genus Staphylococcus was the most abundant genus identified in colostrum samples, independent of quarter, parity, and mastitis status. Furthermore, g_Staphylococcus, g_Fusobacterium, g_Acinetobacter, and g_Bacteroides were more abundant in colostrum samples of multiparous cows than in primiparous cows. The prevalence of Staphylococcus spp. during the periparturient period in primiparous and multiparous cows was investigated by Matthews et al. (1992) and, in agreement with our findings, they found Staphylococcus spp. to be more prevalent in primiparous than in mul- 
tiparous samples (Matthews et al., 1992). A recently published study described differences in PCR-based bacteriological results between samples obtained directly from the udder cistern using a needle and vacuum tube and samples obtained conventionally (Hiitiö et al., 2016). In the conventional samples, the number of positive Staphylococcus spp. was more than twice that of samples taken with the needle technique, indicating that most of the Staphylococcus spp. could have originated from the teat skin or environmental sources. Although in the present study we performed the disinfection procedure correctly, contamination from the teat skin could still have occurred and consequently affected the microbial taxonomic structure described herein. However, the presence of strict anaerobic bacteria, such as g_Fusobacterium, g_Prevotella, g_Bacteroides, and o_Clostridiales, in our study indicates that the complex colostrum microbial community that we described is likely an accurate representation of the colostrum microbiome and not merely a result of teat skin contamination.

Our results showed that CMC samples from primiparous cows were less diverse than NCMC samples from cows of this same parity; however, the same was not the case for multiparous animals. Reduced microbial diversity has been linked to several diseases in humans, such as obesity (Turnbaugh and Gordon, 2009) and bowel disease (Qin et al., 2010); however, higher microbial diversity has been detected in vaginosis (Fredricks et al., 2005) and respiratory tract illnesses (Charlson et al., 2011). Thus, different niches of the body appear to have different bacterial diversity profiles. In our study, the colostrum sampled from primiparous quarters that eventually developed CM was less diverse than the co-
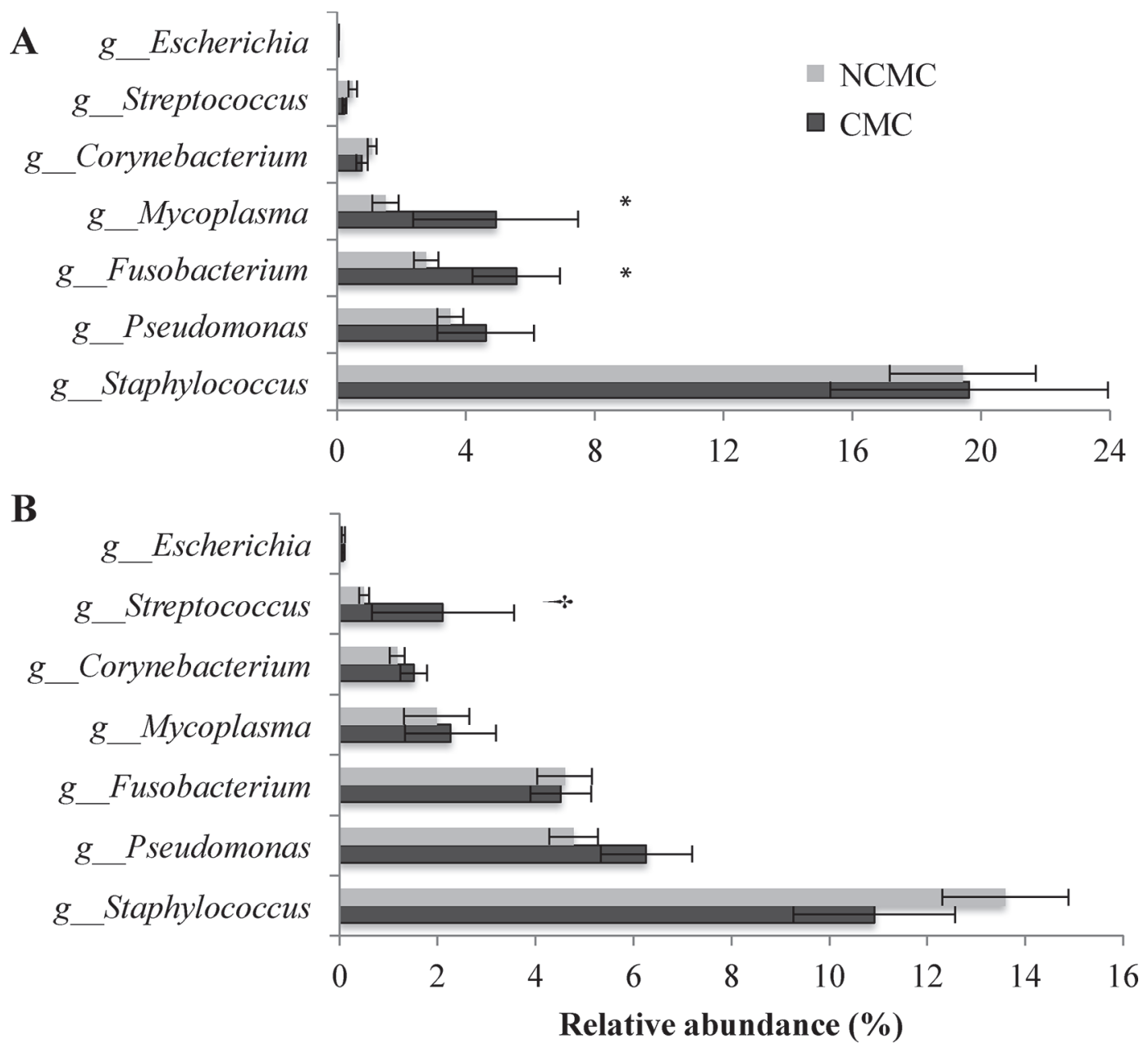

Figure 5. Bar graphs illustrating the mean relative abundance of genera (g) Staphylococcus, Pseudomonas, Fusobacterium, Mycoplasma, Corynebacterium, Streptococcus, and Escherichia detected in the clinical mastitic colostrum (CMC) category (black) and the nonclinical mastitic colostrum (NCMC) category (gray) of primiparous (A) and multiparous (B) cows. Error bars are positioned around the means and represent the SEM. A dagger $(\dagger)$ represents $0.05 \leq P<0.1$ and asterisk $\left(^{*}\right)$ represents $P<0.05$. 
LIMA ET AL.

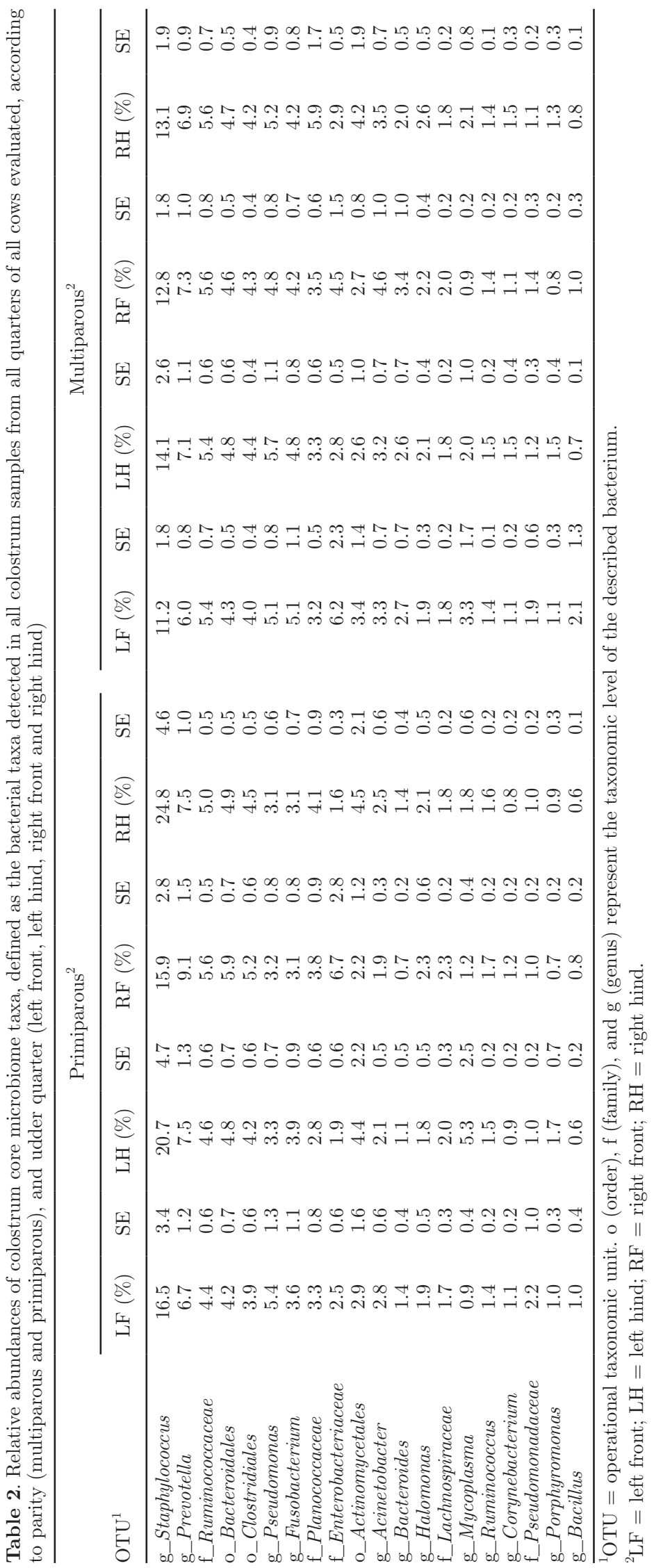


lostrum from quarters that did not experience mastitis during the first $30 \mathrm{~d}$ postpartum, suggesting that, in primiparous colostrum, lower diversity may be associated with future disease events.

At the phylum level, Tenericutes and Fusobacterium were more abundant in CMC than in NCMC of primiparous animals. Both of these phyla contain important bacterial species, such as Mycoplasma spp. (Fox et al., 2005) and Fusobacterium necrophorum (Tan et al., 1996; Oikonomou et al., 2012), which have been associated with the etiopathogenesis of CM. In agreement with the phylum-level results, Mycoplasma and Fusobacterium genera were more abundant in CMC than in NCMC of primiparous cows. Thus, our data suggest that the colostrum microbiome of primiparous animals that eventually developed early CM harbors lower bacterial diversity, and that taxonomic changes occur at the phylum and genus levels associated with mammary gland health; the same was not observed for multiparous cows.

The core microbiome has been described as the population of microbes that is conserved regardless of host genetics and diet, and its disruption could indicate metabolic imbalances and disease of the gut (Ley et al., 2006; Turnbaugh and Gordon, 2009). In our study, the core microbiome was defined as bacterial taxa shared by all colostrum samples of all cows examined, regardless of parity or colostrum category (CMC or NCMC). Twenty taxa were detected as being part of the colostrum core microbiome, including g_Staphylococcus, g_Mycoplasma, and g_Corynebacterium. Some members of these bacterial genera, such as Staphylococcus chromogenes (Pyörälä and Taponen, 2009) and Staphylococcus aureus (Fox et al., 2001), Mycoplasma bovis (Fox and Gay, 1993; Fox et al., 2005; Fox, 2012), and Corynebacterium bovis (Fox and Gay, 1993), have been systematically associated with mastitis. Thus, further studies are needed to better understand the complexity of the colostrum core microbiome and its classification as a taxonomic marker of mammary gland health.

\section{CONCLUSIONS}

We showed that $\mathrm{BC}$ contains a highly diverse and rich microbial community. Primiparous cow colostrum was shown to harbor a richer microbiota than that of multiparous cows, and differences in the microbial community structure between parity were also observed. Differences in diversity and microbial community structure between NCMC and CMC existed for primiparous cows; however, the same was not detected for multiparous cows.

\section{ACKNOWLEDGMENTS}

The present research project was supported by the Agriculture and Food Research Initiative Competitive Grant no. 2013-67015-21233 from the USDA National Institute of Food and Agriculture (Washington, DC).

\section{REFERENCES}

Aarestrup, F. M., and N. E. Jensen. 1997. Prevalence and duration of intramammary infection in Danish heifers during the peripartum period. J. Dairy Sci. 80:307-312.

Addis, M. F., A. Tanca, S. Uzzau, G. Oikonomou, R. C. Bicalho, and P. Moroni. 2016. The bovine milk microbiota: Insights and perspectives from -omics studies. Mol. Biosyst. 12:2539-2372.

Amann, R. I., W. Ludwig, and K. H. Schleifer. 1995. Phylogenetic identification and in situ detection of individual microbial cells without cultivation. Microbiol. Rev. 59:143-169.

Bokulich, N. A., S. Subramanian, J. J. Faith, D. Gevers, J. I. Gordon, R. Knight, D. A. Mills, and J. G. Caporaso. 2013. Quality-filtering vastly improves diversity estimates from Illumina amplicon sequencing. Nat. Methods 10:57-59.

Bradley, A. J., and M. J. Green. 2000. A study of the incidence and significance of intramammary enterobacterial infections acquired during the dry period. J. Dairy Sci. 83:1957-1965.

Bradley, A. J., and M. J. Green. 2004. The importance of the nonlactating period in the epidemiology of intramammary infection and strategies for prevention. Vet. Clin. North Am. Food Anim. Pract. 20:547-568.

Cabrera-Rubio, R., M. C. Collado, K. Laitinen, S. Salminen, E. Isolauri, and A. Mira. 2012. The human milk microbiome changes over lactation and is shaped by maternal weight and mode of delivery. Am. J. Clin. Nutr. 96:544-551.

Caporaso, J. G., C. L. Lauber, W. A. Walters, D. Berg-Lyons, J. Huntley, N. Fierer, S. M. Owens, J. Betley, L. Fraser, M. Bauer, N. Gormley, J. A. Gilbert, G. Smith, and R. Knight. 2012. Ultrahigh-throughput microbial community analysis on the Illumina HiSeq and MiSeq platforms. ISME J. 6:1621-1624.

Capuco, A. V., R. M. Akers, and J. J. Smith. 1997. Mammary growth in Holstein cows during the dry period: quantification of nucleic acids and histology. J. Dairy Sci. 80:477-487.

Chao, A. 1984. Nonparametric estimation of the number of classes in a population. Scand. J. Stat. 11:265-270.

Charlson, E. S., K. Bittinger, A. R. Haas, A. S. Fitzgerald, I. Frank, A. Yadav, F. D. Bushman, and R. G. Collman. 2011. Topographical continuity of bacterial populations in the healthy human respiratory tract. Am. J. Respir. Crit. Care Med. 184:957-963.

Chase, C. C., D. J. Hurley, and A. J. Reber. 2008. Neonatal immune development in the calf and its impact on vaccine response. Vet. Clin. North Am. Food Anim. Pract. 24:87-104.

Edgar, R. C. 2010. Search and clustering orders of magnitude faster than BLAST. Bioinformatics 26:2460-2461.

Elfstrand, L., H. Lindmark-Månsson, M. Paulsson, L. Nyberg, and B. Åkesson. 2002. Immunoglobulins, growth factors and growth hormone in bovine colostrum and the effects of processing. Int. Dairy J. 12:879-887.

Falentin, H., L. Rault, A. Nicolas, D. S. Bouchard, J. Lassalas, P. Lamberton, J. M. Aubry, P. G. Marnet, Y. Le Loir, and S. Even. 2016. Bovine teat microbiome analysis revealed reduced alpha diversity and significant changes in taxonomic profiles in quarters with a history of mastitis. Front. Microbiol. 7:480.

IDF. 1987. Machine Milking and Mastitis. Bull. Int. Dairy Fed. International Dairy Federation, Brussels, Belgium.

Fox, L. K. 2012. Mycoplasma mastitis: Causes, transmission, and control. Vet. Clin. North Am. Food Anim. Pract. 28:225-237.

Fox, L. K., K. W. Bayles, and G. A. Bohach. 2001. Staphylococcus aureus mastitis. Pages 271-294 in Staphylococcus aureus Infection and Disease. Springer, New York, NY. 
Fox, L. K., and J. M. Gay. 1993. Contagious mastitis. Vet. Clin. North Am. Food Anim. Pract. 9:475-487.

Fox, L. K., J. H. Kirk, and A. Britten. 2005. Mycoplasma mastitis: A review of transmission and control. J. Vet. Med. B Infect. Dis. Vet. Public Health 52:153-160.

Fredricks, D. N., T. L. Fiedler, and J. M. Marrazzo. 2005. Molecular identification of bacteria associated with bacterial vaginosis. N. Engl. J. Med. 353:1899-1911.

Gilbert, J. A., F. Meyer, D. Antonopoulos, P. Balaji, C. T. Brown, N. Desai, J. A. Eisen, D. Evers, D. Field, W. Feng, D. Huson, J. Jansson, R. Knight, J. Knight, E. Kolker, K. Konstantindis, J. Kostka, N. Kyrpides, R. Mackelprang, A. McHardy, C. Quince, J. Raes, A. Sczyrba, Shade, and R. Stevens. 2010. Meeting report: The terabase metagenomics workshop and the vision of an earth microbiome project. Stand. Genomic Sci. 3:243-248.

Green, M. J., L. E. Green, G. F. Medley, Y. H. Schukken, and A. J. Bradley. 2002. Influence of dry period bacterial intramammary infection on clinical mastitis in dairy cows. J. Dairy Sci. 85:25892599

Hammon, H. M., J. Steinhoff-Wagner, J. Flor, U. Schönhusen, and C. C. Metges. 2013. Lactation biology symposium: Role of colostrum and colostrum components on glucose metabolism in neonatal calves. J. Anim. Sci. 91:685-695.

Hiitiö, H., H. Simojoki, P. Kalmus, J. Holopainen, S. Pyörälä, and S. Taponen. 2016. The effect of sampling technique on the PCRbased bacteriological results of bovine milk samples. J. Dairy Sci. 99:6532-6541.

Khodayar-Pardo, P., L. Mira-Pascual, M. C. Collado, and C. MartínezCosta. 2014. Impact of lactation stage, gestational age and mode of delivery on breast milk microbiota. J. Perinatol. 34:599-605.

Kuehn, J. S., P. J. Gorden, D. Munro, R. Rong, Q. Dong, P. J. Plummer, C. Wang, and G. J. Phillips. 2013. Bacterial community profiling of milk samples as a means to understand culture-negative bovine clinical mastitis. PLoS One 8:e61959.

Ley, R. E., P. J. Turnbaugh, S. Klein, and J. I. Gordon. 2006. Microbial ecology: Human gut microbes associated with obesity. Nature 444:1022-1023.

Lindner, J. D. D., M. Santarelli, C. T. Yamaguishi, C. R. Soccol, and E. Neviani. 2011. Recovery and identification of bovine colostrum microflora using traditional and molecular approaches. Food Technol. Biotechnol. 49:364.

Matthews, K. R., R. J. Harmon, and B. E. Langlois. 1992. Prevalence of Staphylococcus species during the periparturient period in primiparous and multiparous cows. J. Dairy Sci. 75:1835-1839.

McDonald, D., M. N. Price, J. Goodrich, E. P. Nawrocki, T. Z. DeSantis, A. Probst, G. L. Andersen, R. Knight, and P. Hugenholtz. 2012. An improved Greengenes taxonomy with explicit ranks for ecological and evolutionary analyses of bacteria and archaea. ISME J. 6:610-618.

Natzke, R. P. 1981. Elements of mastitis control. J. Dairy Sci. 64:14311442.

NMC. 1999. Laboratory Handbook on Bovine Mastitis. National Mastitis Council, New Prague, MN.

NRC. 2001. Nutrient Requirements of Dairy Cattle. Natl. Acad. Press, Washington, DC.

Ogra, S. S., D. Weintraub, and P. L. Ogra. 1977. Immunologic aspects of human colostrum and milk. III. Fate and absorption of cellular and soluble components in the gastrointestinal tract of the newborn. J. Immunol. 119:245-248.

Oikonomou, G., M. L. Bicalho, E. Meira, R. E. Rossi, C. Foditsch, V. S. Machado, A. G. Teixeira, C. Santisteban, Y. H. Schukken, and R. C. Bicalho. 2014. Microbiota of cow's milk; distinguishing healthy, sub-clinically and clinically diseased quarters. PLoS One 9:e85904.

Oikonomou, G., V. S. Machado, C. Santisteban, Y. H. Schukken, and R. C. Bicalho. 2012. Microbial diversity of bovine mastitic milk as described by pyrosequencing of metagenomic $16 \mathrm{~s}$ rDNA. PLoS One $7: \mathrm{e} 47671$

Oviedo-Boyso, J., J. J. Valdez-Alarcón, M. Cajero-Juárez, A. OchoaZarzosa, J. E. López-Meza, A. Bravo-Patiño, and V. M. Baizabal-Aguirre. 2007. Innate immune response of bovine mammary gland to pathogenic bacteria responsible for mastitis. J. Infect. $54: 399-409$

Pakkanen, R., and J. Aalto. 1997. Growth factors and antimicrobial factors of bovine colostrum. Int. Dairy J. 7:285-297.

Pyörälä, S., and S. Taponen. 2009. Coagulase-negative staphylococciemerging mastitis pathogens. Vet. Microbiol. 134:3-8.

Qin, J., R. Li, J. Raes, M. Arumugam, K. S. Burgdorf, C. Manichanh, T. Nielsen, N. Pons, F. Levenez, T. Yamada, D. R. Mende, J. Li, J. Xu, S. Li, D. Li, J. Cao, B. Wang, H. Liang, H. Zheng, Y. Xie, J. Tap, P. Lepage, M. Bertalan, J. M. Batto, T. Hansen, D. Le Paslier, A. Linneberg, H. B. Nielsen, E. Pelletier, P. Renault, T. Sicheritz-Ponten, K. Turner, H. Zhu, C. Yu, M. Jian, Y. Zhou, Y. Li, X. Zhang, N. Qin, H. Yang, J. Wang, S. Brunak, J. Doré, F. Guarner, K. Kristiansen, O. Pedersen, J. Parkhill, J. Weissenbach, P. Bork, S. D. Ehrlich, and M. Consortium. 2010. A human gut microbial gene catalogue established by metagenomic sequencing. Nature 464:59-65.

Rasolofo, E. A., D. St-Gelais, G. LaPointe, and D. Roy. 2010. Molecular analysis of bacterial population structure and dynamics during cold storage of untreated and treated milk. Int. J. Food Microbiol. 138:108-118.

Rollin, E., K. C. Dhuyvetter, and M. W. Overton. 2015. The cost of clinical mastitis in the first 30 days of lactation: An economic modeling tool. Prev. Vet. Med. 122:257-264.

Schloss, P. D., and J. Handelsman. 2005. Introducing DOTUR, a computer program for defining operational taxonomic units and estimating species richness. Appl. Environ. Microbiol. 71:1501-1506.

Tan, Z. L., T. G. Nagaraja, and M. M. Chengappa. 1996. Fusobacterium necrophorum infections: virulence factors, pathogenic mechanism and control measures. Vet. Res. Commun. 20:113-140.

Taponen, S., L. Salmikivi, H. Simojoki, M. T. Koskinen, and S. Pyörälä. 2009. Real-time polymerase chain reaction-based identification of bacteria in milk samples from bovine clinical mastitis with no growth in conventional culturing. J. Dairy Sci. 92:26102617.

Turnbaugh, P. J., and J. I. Gordon. 2009. The core gut microbiome, energy balance and obesity. J. Physiol. 587:4153-4158.

USDA. 2007. Dairy 2007: Biosecurity Practices on U.S. Dairy Operations. USDA. Animal and Plant Health Inspection Service, Veterinary Services, Center for Epidemiology and Animal Health, Fort Collins, CO

Ward, T. L., S. Hosid, I. Ioshikhes, and I. Altosaar. 2013. Human milk metagenome: A functional capacity analysis. BMC Microbiol. $13: 116$

Watters, R. D., J. N. Guenther, A. E. Brickner, R. R. Rastani, P. M. Crump, P. W. Clark, and R. R. Grummer. 2008. Effects of dry period length on milk production and health of dairy cattle. J. Dairy Sci. 91:2595-2603.

Zand, S. M. 1976. Indexes associated with information theory in water quality. J. Water Pollut. Control Fed. 48:2026-2031. 